\title{
Restoring stone monuments: Enlightening critical details by the combined use of innovative sensing techniques
}

\author{
Ermioni D. Pasiou \\ Laboratory for Testing and Materials, Zografou Campus, National Technical University of Athens, Greece \\ Acropolis Restoration Service (YSMA), Ministry of Culture and Sports, Athens, Greece \\ epasion@teemail.gr
}

\begin{abstract}
The experience gathered during the implementation of long series of laboratory experimental protocols, aiming to study the mechanical response of restored structural elements, is summarized. Conclusions are drawn concerning the proper exploitation of the laboratory results in field applications in the frame of on-going restoration projects of ancient stone monuments. The need of continuous bidirectional interaction between the scientific personnel working in the site and the scientists working in the laboratory is emphasized. The role of the Digital Image Correlation technique in quantifying parasitic effects influencing the laboratory data is proven decisive. The need to use modern sensing techniques, providing data from the interior of loaded restored complexes (simulating restored structural elements), like the Pressure Stimulated Currents and the Acoustic Emissions ones, according to a combined manner is highlighted. The capability of these two techniques to monitor the damage evolution within the mass of the elements tested and to provide clear pre-failure indicators renders them flexible tools in the hands of engineers designing the restoration projects.
\end{abstract}

KEYWORDS. 3D digital image correlation; Acoustic emissions; Pressure stimulated currents; Dionysos marble; Monuments.

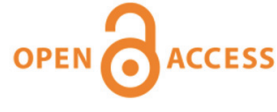

Citation: Pasiou, E. D., Restoring stone monuments: Enlightening critical details by the combined use of innovative sensing techniques, Frattura ed Integrità Strutturale, 50 (2019) 560-572.

Received: 26.01.2019

Accepted: 29.05.2019 Published: 01.10.2019

Copyright: (C) 2019 This is an open access article under the terms of the CC-BY 4.0, which permits unrestricted use, distribution, and reproduction in any medium, provided the original author and source are credited.

\section{INTRODUCTION}

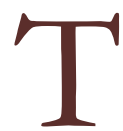
he restoration of monuments of Cultural Heritage is a demanding project since each monument is unique with its own special needs and requirements. The monuments of the Acropolis of Athens are among the worldwide known monuments under restoration. They were built in the $5^{\text {th }}$ century BC. Pentelic marble blocks were used for the construction without any binding material between the surfaces of the blocks. The structural elements (marble volumes) were connected to each other with "I"-shaped connectors and dowels made of layers of soft pure iron and tough steel [1,2], which were placed in mortises (grooves) of suitable geometry. After the placement of the metallic elements the grooves were filled with molten lead [3]. 
Due to the extensive failures observed on the monuments of the Acropolis, caused by a series of disasters (either due to human interventions or natural phenomena), the Greek Ministry of Culture founded the "Committee for the Conservation of the Acropolis Monuments" (ESMA) in 1975 and the "journey" of restoring the monuments of the Acropolis started. At the very beginning of the restoration project, it was observed that the vast majority of the connections (i.e., the "connector-intermediate material-marble surrounding the groove" complex) were destroyed: Either the connectors were fractured and/or the surrounding marble volume was fragmented. In this context, the engineers in charge confronted a series of problems and pressing questions that had to be answered:

1. The use of iron during previous restoration projects was almost exclusively responsible for the fractures. Indeed, iron was oxidized, the connectors swelled and the surrounding marble was fractured. What type of metal could substitute iron for the construction of the new connectors?

2. Would the chosen metal affect the intermediate material in the grooves? If the answer was "yes", which material would it be suitable and compatible?

3. Mining of marble volumes from mount Pentelicon was forbidden. What type of marble was compatible with the ancient one and could be used for the construction of supplements or new blocks?

To answer the aforementioned questions, the engineers in charge for the restoration project collaborated with scientists from various universities and disciplines in order to thoroughly study the mechanical and chemical properties of the ancient materials and the candidate substitute ones. After a long period of intensive study, it was concluded that the proper substitute materials are the following:

1. Grade 2 pure titanium instead of iron $[4,5]$.

2. White cement mortar instead of molten lead [6], taking into account that lead and titanium produce galvanic element.

3. Dionysos marble instead of Pentelic marble [7-9].

Therefore, during the restoration project in progress of the Parthenon, the marble blocks are connected to each other with titanium connectors and the groove is filled with suitable mortar.

The mechanical behaviour of the as above designed horizontal connections, i.e., those with "I"-shaped connectors, was one of the problems studied in-situ by the restoration personnel, almost at the beginning of the restoration project. More specifically, their response under tensile loading was studied experimentally [10]. However, the "I"-shaped connectors are also stressed under shear (observations of the members of the temple in-situ supports the shear loading of the connectors [10]), but the problem had not been investigated until recently. The study of the response of the new design of the connectors under shear started around 2008, in the Laboratory for Testing and Materials (LTM) of the National Technical University of Athens (NTUA). A series of problems, usually coming up during experimental protocols, appeared and had to be solved as it is discussed in next sections.

\section{THE SPECIMENS AND THE EXPERIMENTAL SET-UP}

$\mathrm{D}$ ionysos marble is an orthotropic, extremely brittle material [7-9]. Its mechanical response is characterized by an extremely pronounced "size effect" [11-13] dictating the use of large specimens (the dimensions of which should ideally approach those of actual structural members) in order for the results obtained experimentally to be size independent [14,15]. Simulating a typical connection of the epistyles of the Parthenon Temple under a 1:3 scale (and at the same time of marble blocks of the entablature of the temple under a 1:1 scale), the minimum length of the specimens was estimated to about $50 \mathrm{~cm}$ (note that the scales refer to the dimensions of the connector and the groove). The dimensions of the marble volumes, especially the distance between the web of the groove and the free surface of the blocks, were based on in-situ observations [16].

The next question to be answered was related to the exact geometry of the specimens that would enable the implementation of pure shear experiments. How should the specimen be gripped and loaded? In which loading frame of a typical laboratory could the experiments be carried out? Based on the experience of the personnel of the restoration project, it was decided to join together two Dionysos marble blocks with one titanium "I"-shaped connector (Fig.1a) and mortar. The main difference between the specimens of previous protocols [17] and the protocol here described was the shape of the marble volumes. Indeed, in previous protocols (and in the preliminary stage of the protocol here described) both volumes were prismatic (Fig.1b) and the smaller one was fixed on the table of the loading frame using a series of rigid metallic plates, rods and nuts. Two holes were drilled through the "thickness" of the larger volume enabling loading of the specimen with the aid of a couple of rigid bars. That experimental set-up is shown in Fig.2a and more details can be found in [17].

On the other hand, according to the new design of the specimens, only one of the two volumes was prismatic while the second one was " $\Gamma$ "-shaped (Fig.1c). The prismatic volume was again fixed on the table of the loading frame, while on the 
" $\Gamma$ '-shaped one (Fig.1c) two through-the-thickness holes were drilled symmetrically with respect to the volumes' interface. In other words, the load was imposed symmetrically with respect to the marbles' interface avoiding parasitic moments [18]. Two groups of specimens with the specific geometry were tested: (a) Group A: The groove is completely filled with the mortar used in the restoration project (right sketch of Fig.1d) and, (b) Group B: The groove is filled with the typical mortar but a small portion of the groove (on both sides of the marble volumes' interface) remains uncovered, i.e., the central part of the connector is visible. This area is called "relieving space" (left sketch of Fig.1d) and it is adopted during the restoration project after Dr Zambas' suggestion [10].

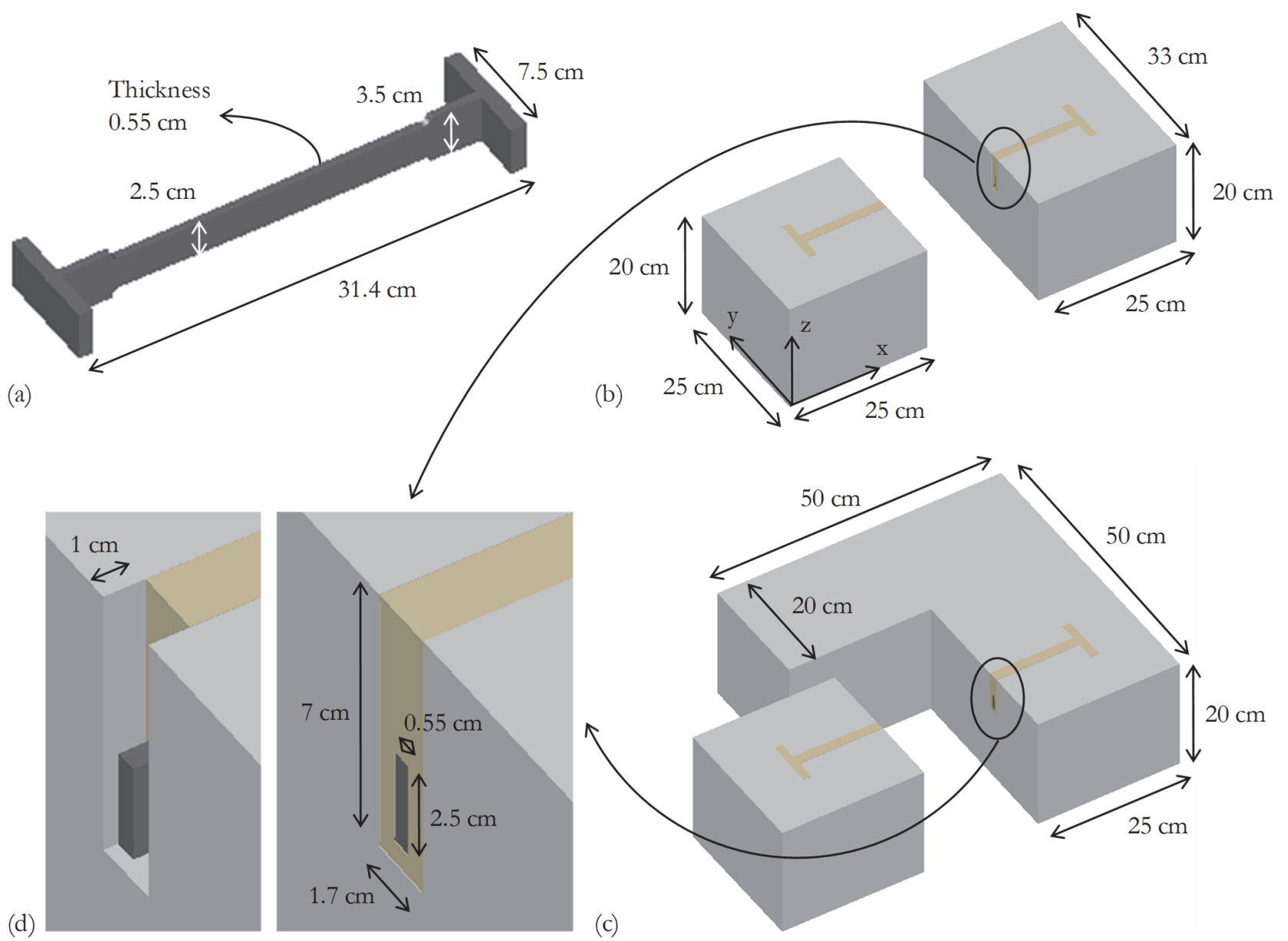

Figure 1: (a) Titanium connector; The specimens of (b) previous protocols and (c) the experimental protocol here described; (d) Detail of the central area of the specimens (where both the mortar and the connector are shown).

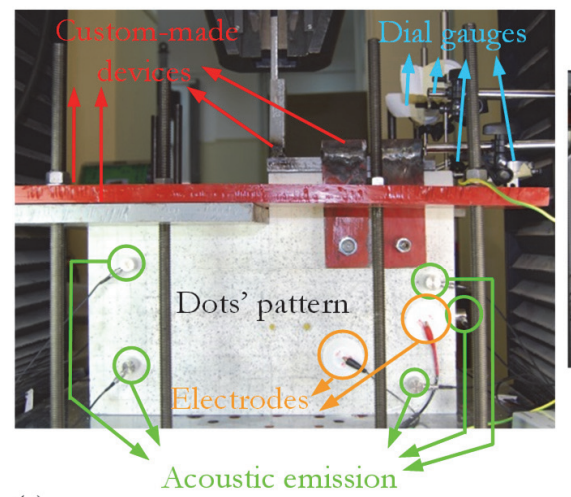

(a)

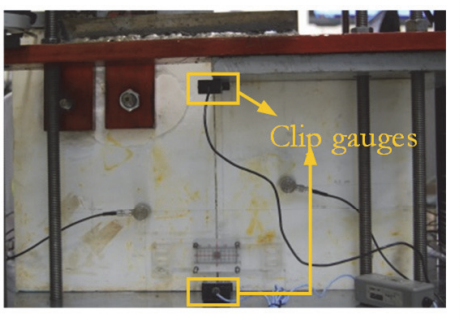

(b)
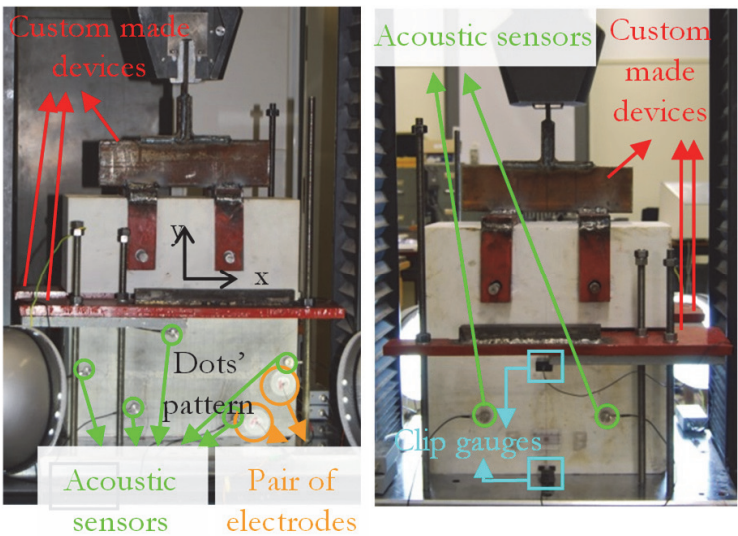

sensors electrodes

Figure 2: The experimental set-up of the (a) preliminary [17] and (b) main [18] experimental protocol. 


\section{HIDDEN DETAILS ENLIGHTENED USING THE DIGITAL IMAGE CORRELATION TECHNIQUE}

he actual displacement of the moving marble volume was determined with the aid of the Digital Image Correlation (DIC) technique and it was validated against the data of traditional dial gauges. It was found significantly different compared to the displacement of the traverse of the loading frame (Fig.3a). Of course, the use of only dial gauges could be enough for the determination of the displacement of the movable block. However, the DIC technique (in its three dimensional version) was considered necessary due to its capability to determine the full-field displacement components, i.e., all over the surface of the specimen monitored. In Fig.3b the full-field displacement components of a typical specimen of Group A of the main experimental protocol is shown. The surface of the specimen monitored by the cameras of the DIC is the one with the groove and from now on this surface will be called "front" surface of the specimen. It should be mentioned that the sensitivity of the system depends on the size of the "optical window" (field of view). Before each experiment, the user of the DIC technique must decide between larger "optical window" with lower sensitivity and smaller "optical window" with higher sensitivity, based on the specific needs of each test.
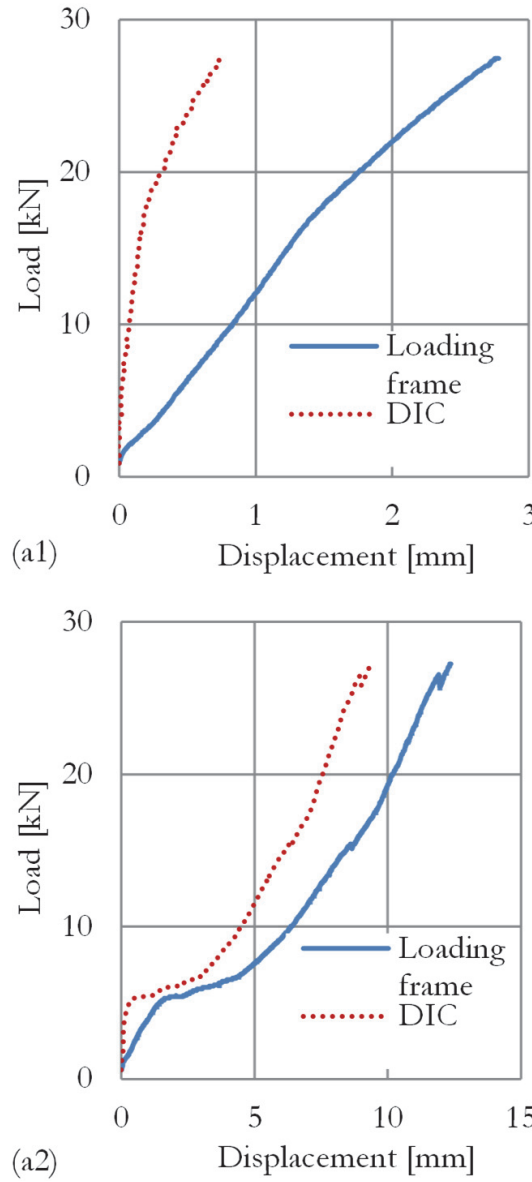
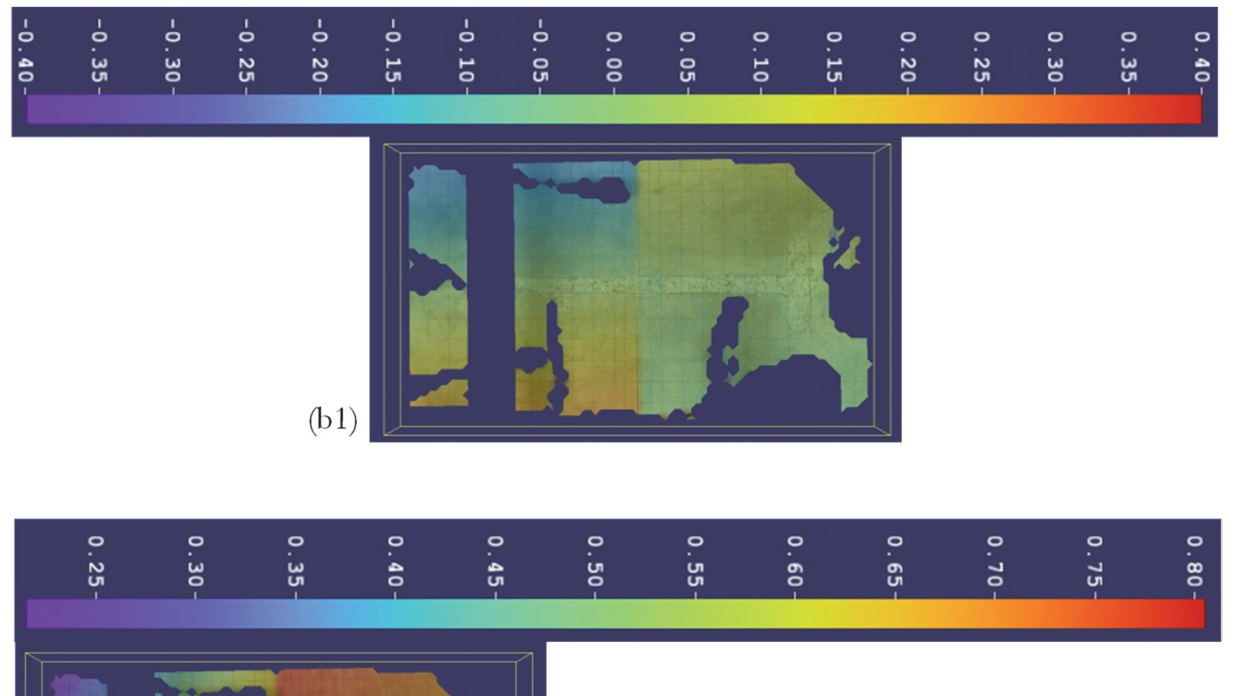

(b2)

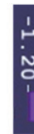

(b3)

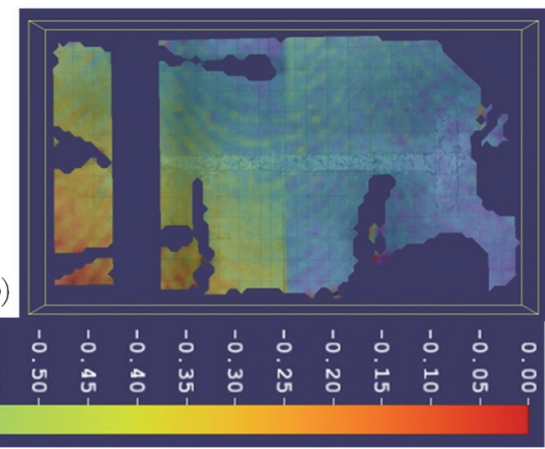

Figure 3: Load-displacement curves for a typical specimen of (a1) Group A and (a2) Group B of the main experimental protocol; The displacement field for a typical specimen of Group A along the (b1) x-axis, (b2) y-axis and (b3) z-axis (the reference system is shown in Fig.1).

In any case, if the full-field displacement components are determined, the researcher is able to study the deformation at any point or along any line or even over an area of the specimen monitored. The fact that the selection of these points/lines/ areas can be made after the experiment (post-mortem) is one of the most advantageous characteristics of the DIC technique. Following the procedure just described for a typical specimen consisting of two prismatic marble volumes (during previously implemented protocols [12] and, also, during the preliminary stage of the present one), two small areas of the mortar's surface were isolated on both sides of the marble volumes' interface (green and blue rectangles in Fig.4(a1) for the moving and the fixed volume, respectively). The variation of their horizontal displacements was obtained (Fig.4(a2) where the same colour code is used). Based on these graphs, it was concluded that the mortar was fractured (i.e., the two mortar surfaces 
started moving apart from each other) at a load-level equal to about $10 \mathrm{kN}$. This time instant corresponds to a load drop observed at the respective load-time curve, confirming the failure of the mortar. In addition, during the preliminary stage of the protocol, the DIC technique permitted quantification of the parasitic bending tendency of the moving block of the specimens as it can be seen in Fig.4b. In this figure the horizontal displacement recorded at the lower level of the moving block is presented for some characteristic load levels. The latter was the catalytic reason for the design and construction of specimens with different geometry, used during the main stage of the present experimental protocol.

In all experiments of this stage, the moving " $\Gamma$ "-shaped volume was displaced almost perfectly parallel to the fixed one (i.e., along their interface), as it was confirmed by the DIC technique. The horizontal displacements (along the length of the connector) on the "front" surface of a typical specimen of Group A, just before its fracture, are presented in Fig.3(b1). The blank areas of the figure are the areas of the specimen on which various sensors were attached and also the areas of the specimen which are hidden by the metallic rods used for the fixation of the prismatic volume on the table of the loading frame (see Fig.2b). It is seen from Fig.3(b1) that the field of horizontal displacements is almost uniform all over the moving volume. The respective maximum values do not exceed $0.3 \mathrm{~mm}$, as it is concluded by the variation of the opening/closing (displacement along the x-axis) of the blocks during the whole duration of the experiment (Fig.5a). It is to be noted that the displacements presented in Fig.5a were measured at the upper and lower level of the blocks' interface.

It could be anticipated that a three dimensional motion cannot be fully described in terms of the displacements of a single plane (in this case the front surface of the specimen). In this context, additional data are required concerning the displacements of a second material plane. For this reason, two clip gauges were attached on the rear surface of the specimen (at the aforementioned levels of the volumes' interface). However, it is to be mentioned that clip gauges provide data concerning exclusively the displacement which is parallel to the knife edges (on which the clip gauges are attached). In other words, they only record the "normal" displacement of the volumes (i.e., perpendicularly to the blocks' interface), which is plotted in Fig.5b for a typical specimen of Group A (recall that the respective displacement on the front surface of the same specimen were presented in Fig.3(b1) and Fig.5a).

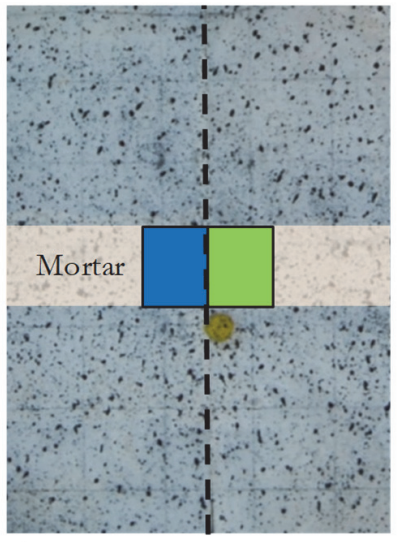

(a1) Epistyles' interface

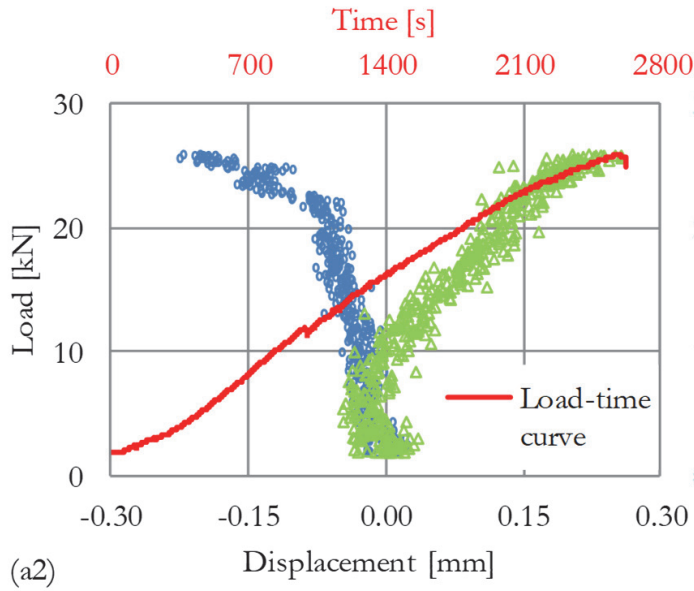

(a2)

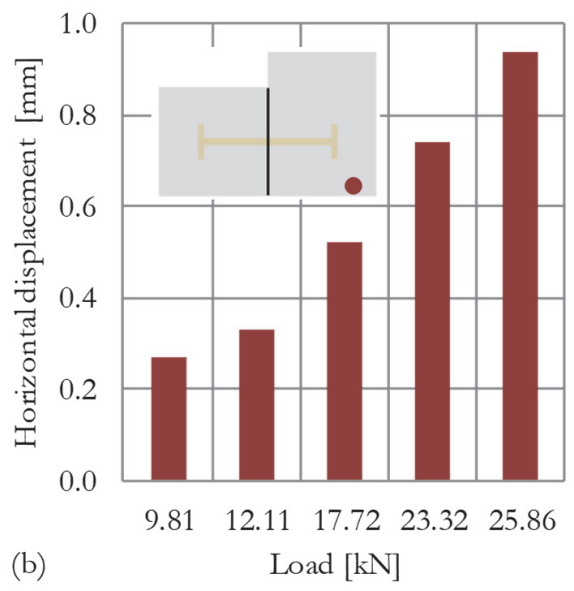

(b)

Figure 4: (a1) Enlarged view of the marble volumes' interface around the mortar (at the center of a typical specimen of the preliminary protocol); (a2) The horizontal displacements of the two isolated mortar surfaces (shown in (a1)) versus the applied load; (b) The horizontal displacement of the lower level of the moving block for various loads. The measurements are referred to the point of the red circle presented in the embedded figure.

\section{DATA OBTAINED FROM THE INTERIOR OF THE CONNECTION USING A SERIES OF SENSORS/TECHNIQUES}

A lthough the importance of the raw data measured from the external surface of the specimens is not to be disputed, it is nowadays accepted that data must be, also, obtained from the interior of the specimens given that failure mechanisms leading to final fracture are firstly activated within the mass of the specimens. Therefore, the need of sensing systems that can detect the internal damages and monitor its evolution becomes imperative.

\section{Using strain gauges "inside" the specimens}

Strain gauges, attached on the outer surface of the specimens, are the sensors most widely used for measuring strain. However, in some of the experiments of the present study, strain gauges were glued inside the specimens, taking advantage of the 

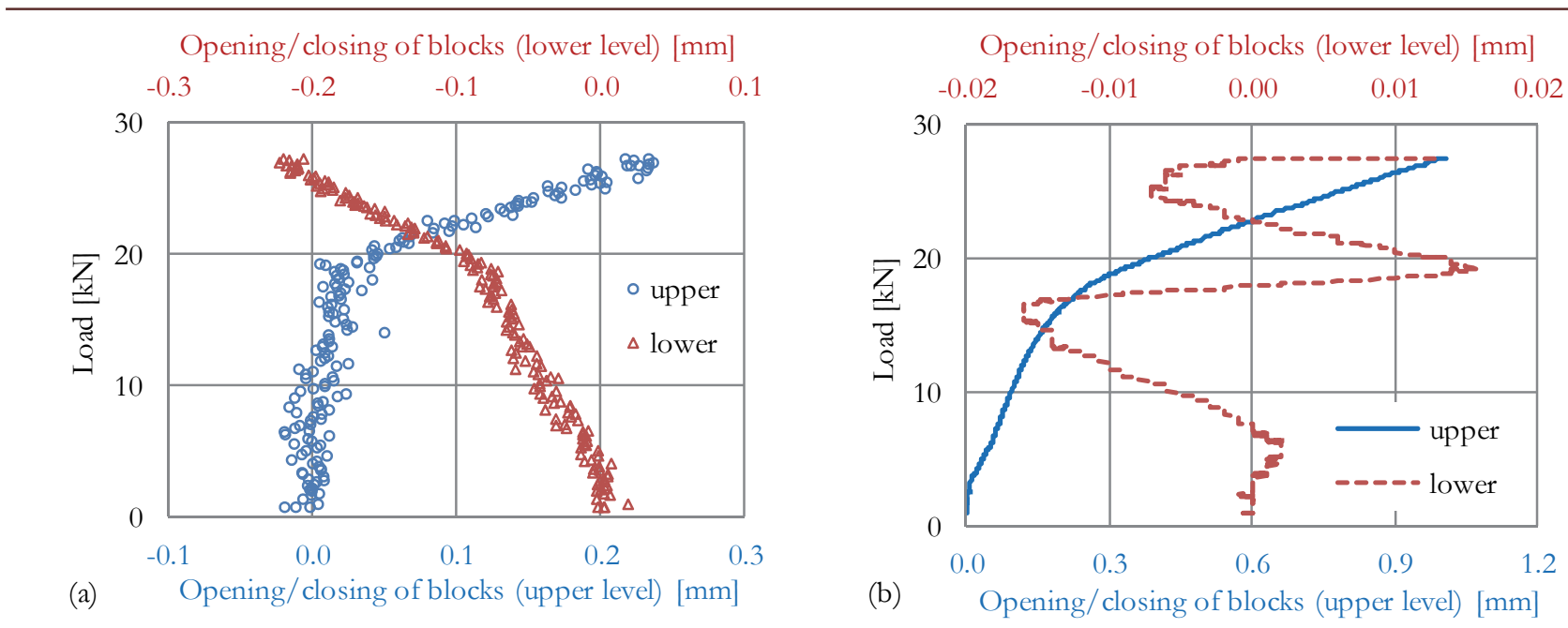

Figure 5: The opening/closing of the marble blocks measured at two height levels of the blocks by (a) DIC technique on the front surface of the specimen and (b) two clip gauges on the back surface of the specimen.

interfaces due to the co-existence of the three materials. More specifically, the strain gauges were properly attached at the central part of the connector (Fig.6a) which is the most severely loaded part. Special attention was paid for the insulation of the strain gauges and their cables, against the moisture of the mortar, using silicone. However, this was not always successful and in some cases the respective electric circuits were interrupted. In Fig.6b the strains recorded at the center of the connector in a typical specimen of Group A is plotted versus the applied load. It is seen from this figure that the central part of the connector is initially under compression while after the load reaches $17.5 \mathrm{kN}$ the magnitude of the compressive strain starts decreasing attaining finally positive values until the end of the experiment. The time instant of this alteration corresponds to the slope change observed in the respective load-time curve.

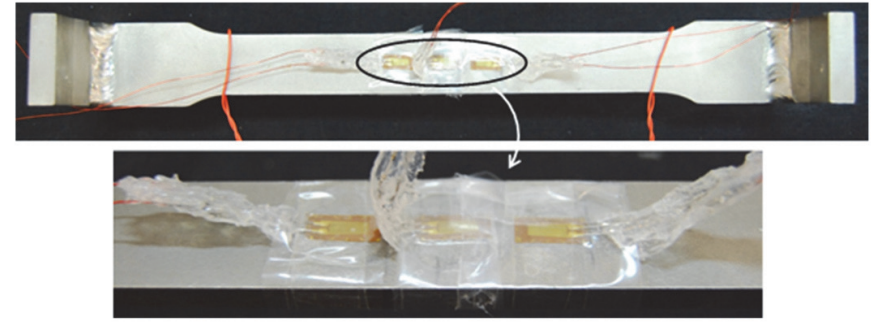

(a)

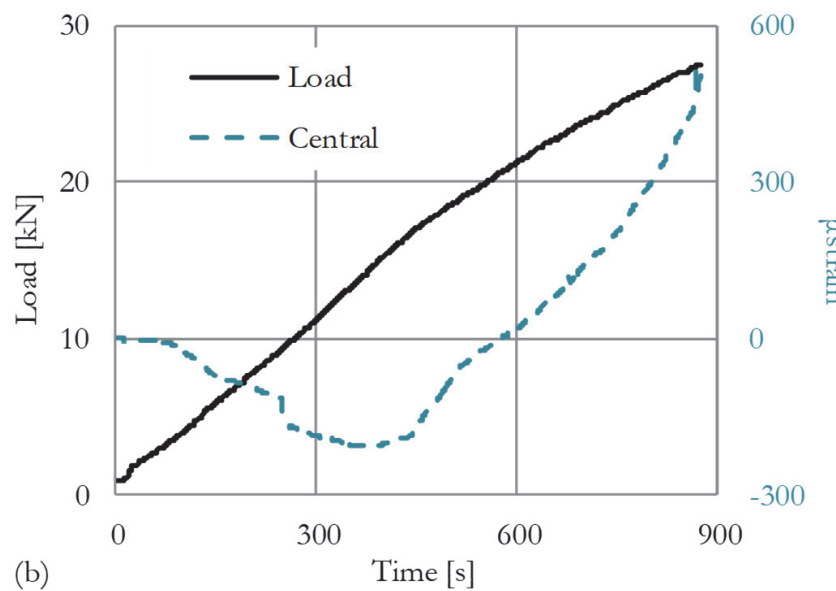

(b)

Figure 6: (a) The strain gauges attached on the connector; (b) The time variation of the strains during a test with a specimen of Group A.

\section{The role of the Acoustic Emissions (AE)}

One of the most common and widely used techniques, the sensors of which are attached on the outer surface of the specimen, providing, however, data for the internal damage of the specimen tested, is the Acoustic Emission (AE) technique. It is a mature, well-established technique used both in laboratory experiments and, also, in the field. One of its most important advantages is the determination of the location of the acoustic events, or in other words of the internal damage of the speci$\mathrm{men} /$ structural member, based on the waves' propagation velocity through the material tested. At this point, the following questions arise: Is the determination of the location of the damage accurate in case of anisotropic materials? Is the determination of the location of the damage accurate in case specimens or structural elements made of more than one material are tested? Obviously, taking into account that one wave velocity is usually considered, the results obtained cannot be accepted without further validation. However, during the last years, two wave velocities can be considered in the AE software and therefore the location accuracy is clearly improved even in case of anisotropic materials, e.g., marble specimens [19]. 
It is recalled that the specimens studied here are made of three completely different materials (anisotropic brittle marblemortar-isotropic ductile titanium) and also three material interfaces exist, i.e., between the connector and mortar, between mortar and marble, and between the two marble blocks. Therefore, the two wave velocities considered definitely improved the determination of the location of the damage. Having determined the location of the damage and taking, also, into account the time instant at which the damage occurs, monitoring the real time damage evolution becomes feasible. Typical images of the damage evolution are presented in Fig.7 for a typical specimen of Group B of the main experimental protocol.

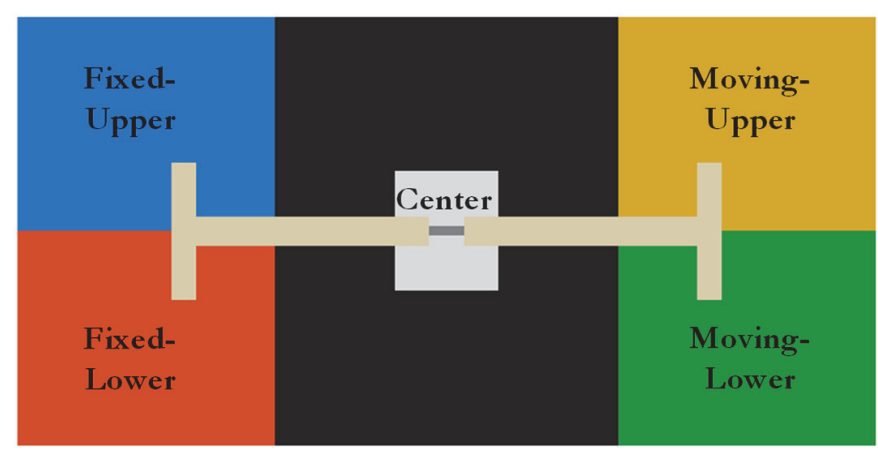

(a)
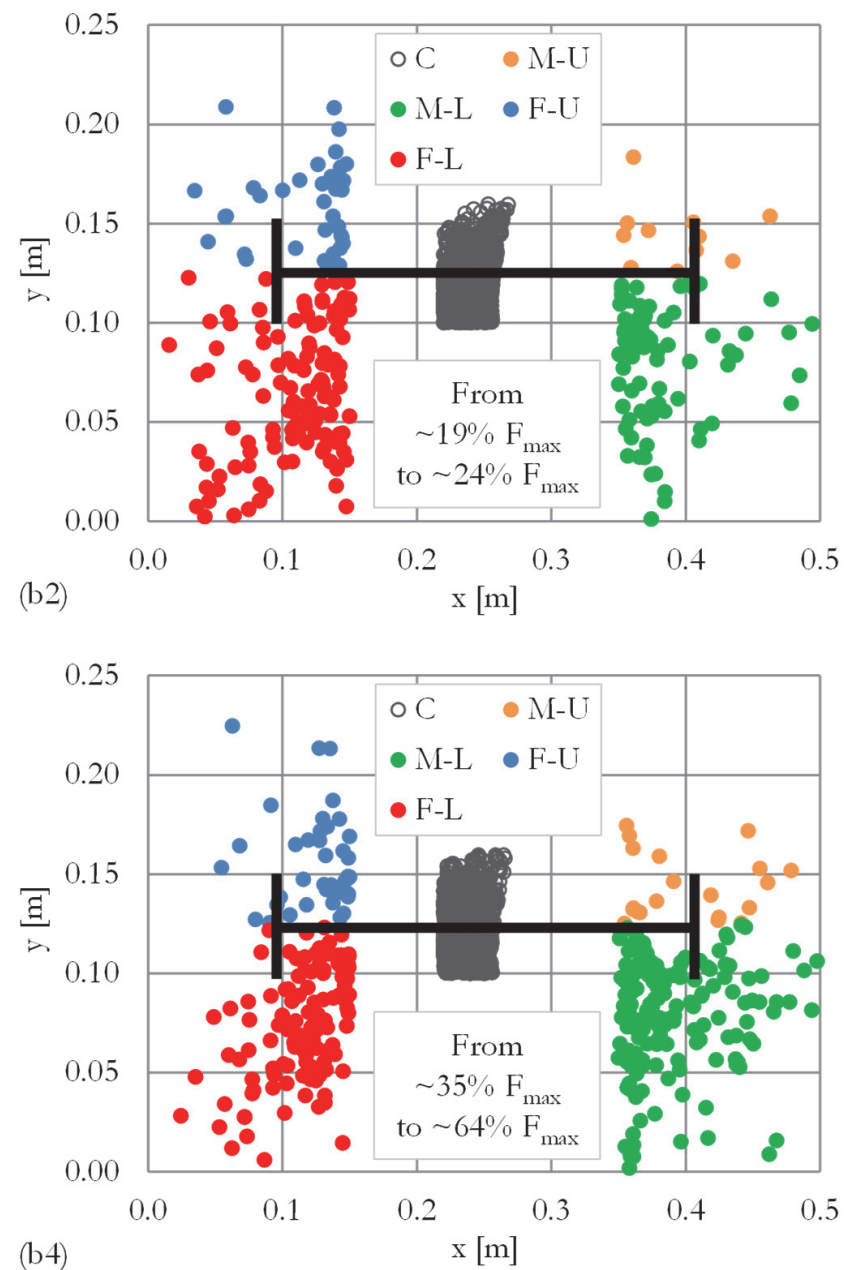
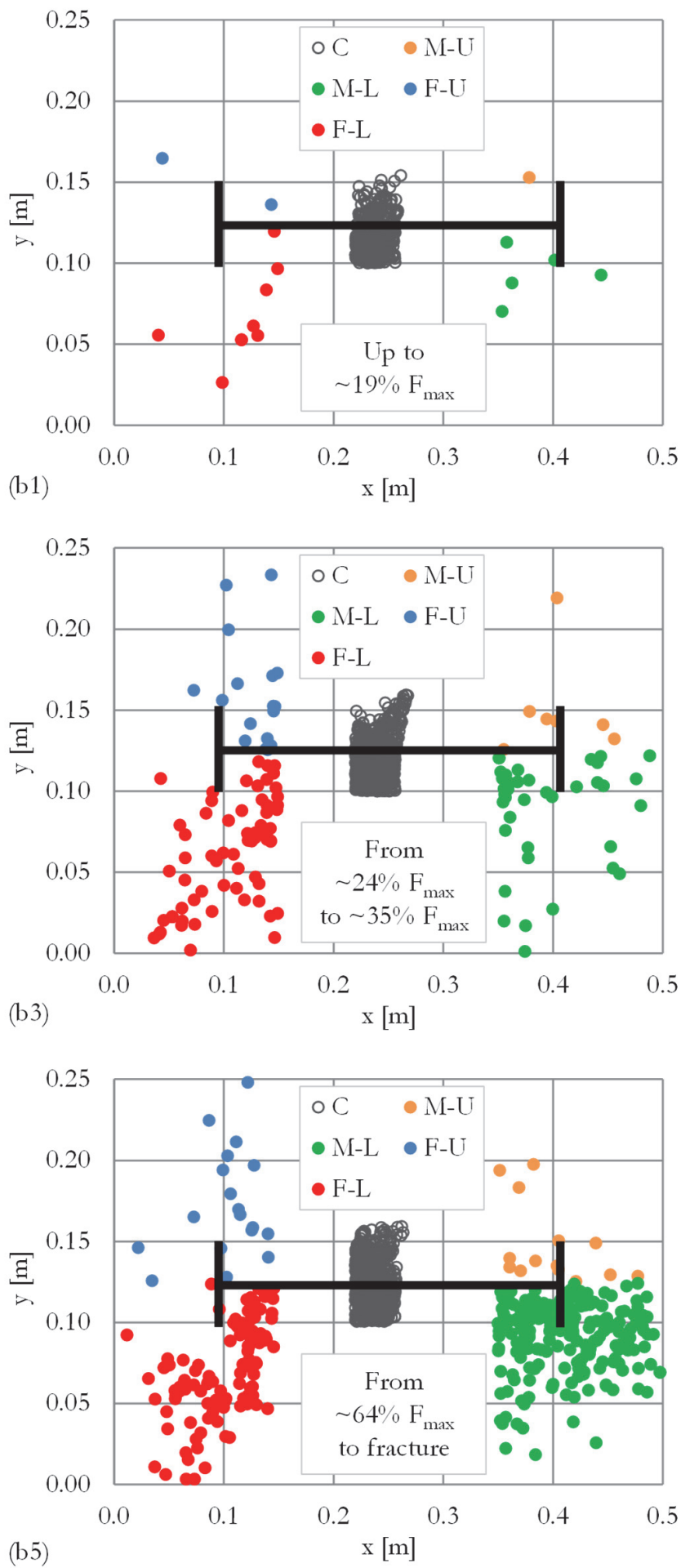

(b5)

Figure 7: (a) The sub-volumes of a typical specimen of Group B; (b) The spatiotemporal distribution of the acoustic events in the subvolumes. The colours of the events correspond to the respective coloured sub-volume. 
The specimen was divided in five "sub-volumes", i.e., the central part of the specimen (grey area in Fig.7a) and four subvolumes around the flanges of the connector (orange, green, red and blue areas in Fig.7a). The black area of the specimen was not taken now into account in order to exclude the acoustic events due to friction between the marble volumes. The specific experiment was divided in five phases based on the slope changes of the respective load-displacement curve (Fig. $3(\mathrm{a} 2))$. As it was expected, and besides the existence of the relieving space, the central part of the specimen is the most severely stressed one even from the very low loading levels (Fig.7(b1)). On the contrary, the damage around the flanges starts becoming severe after about $20 \%$ of the fracture load (Fig.7(b2)). From this point on, the lower part of the specimen is systematically more severely stressed than the upper one. During the last phase of loading (Fig.7(b5)) their quantitative difference is obvious and the number of acoustic events produced in the lower part of the moving block is larger than the respective number produced in all other sub-volumes. Based on Fig.7, it is clear that the damage starts from the center of the specimen and propagates towards the flanges of the connector/groove while the damage is more intense on the moving volume which was indeed fractured finally (in good accordance with previous similar experiments [18]).

Another way of elaborating the raw acoustic data, which is commonly used by researchers to monitor the damage, is the rate of the acoustic hits produced. Recently, an alternative way of representing this quantity was introduced by Triantis and Kourkoulis [20], in terms of the so-called F-function. This function is based on the inter-event times between successive hits and it is usually plotted versus the $\left(t_{\mathrm{f}}-\mathrm{t}\right)$ parameter (where $t_{\mathrm{f}}$ is the time of the specimen's fracture) in a semi-logarithmic graph. The advantage of this representation is the smoother plots obtained, since the time step is usually less than one second. This is crucial especially during the very last seconds before fracture, since it allows a "magnified" view of the acoustic activity just before the specimen's catastrophic failure. In Fig.8 the F-function, determined using the data of two acoustic sensors (one on the moving and one on the fixed block - green and red curve, respectively), is presented for a typical specimen of Group B in juxtaposition to the applied load. The F-function was calculated using 50 successive hits from each sensor. The two peaks observed (at about $1155 \mathrm{~s}$ and $163 \mathrm{~s}$ before the final fracture) correspond to the two load drops (at load levels equal to about $55 \%$ and $97 \%$ of the fracture load, respectively). After the last peak, the acoustic activity recorded by both sensors is quite similar but $30 \mathrm{~s}$ before the fracture the F-functions of the two sensors start diverging from each other. The F-function of the moving block (green curve) starts increasing smoothly until $1.5 \mathrm{~s}$ before the end of the experiment and therefore increases significantly until the specimen's fracture. On the contrary, the acoustic activity in the fixed volume (red curve) remains almost constant. It is to be mentioned that the $\mathrm{x}$-axis of Fig. 8 is the mean value of the $\left(t_{\mathrm{f}}-\mathrm{t}\right)$ parameters of the respective 50 hits. The fact that the last point of the green curve corresponds to $\left(t_{\mathrm{f}}-\mathrm{t}\right)=0.16 \mathrm{~s}$ while that of the red curve corresponds to $\left(t_{\mathrm{f}}-\mathrm{t}\right)=1.8 \mathrm{~s}$ denotes that the last 50 hits in the moving block are produced within a very short time interval while in the fixed block the acoustic activity as represented by the number of hits is sparse.

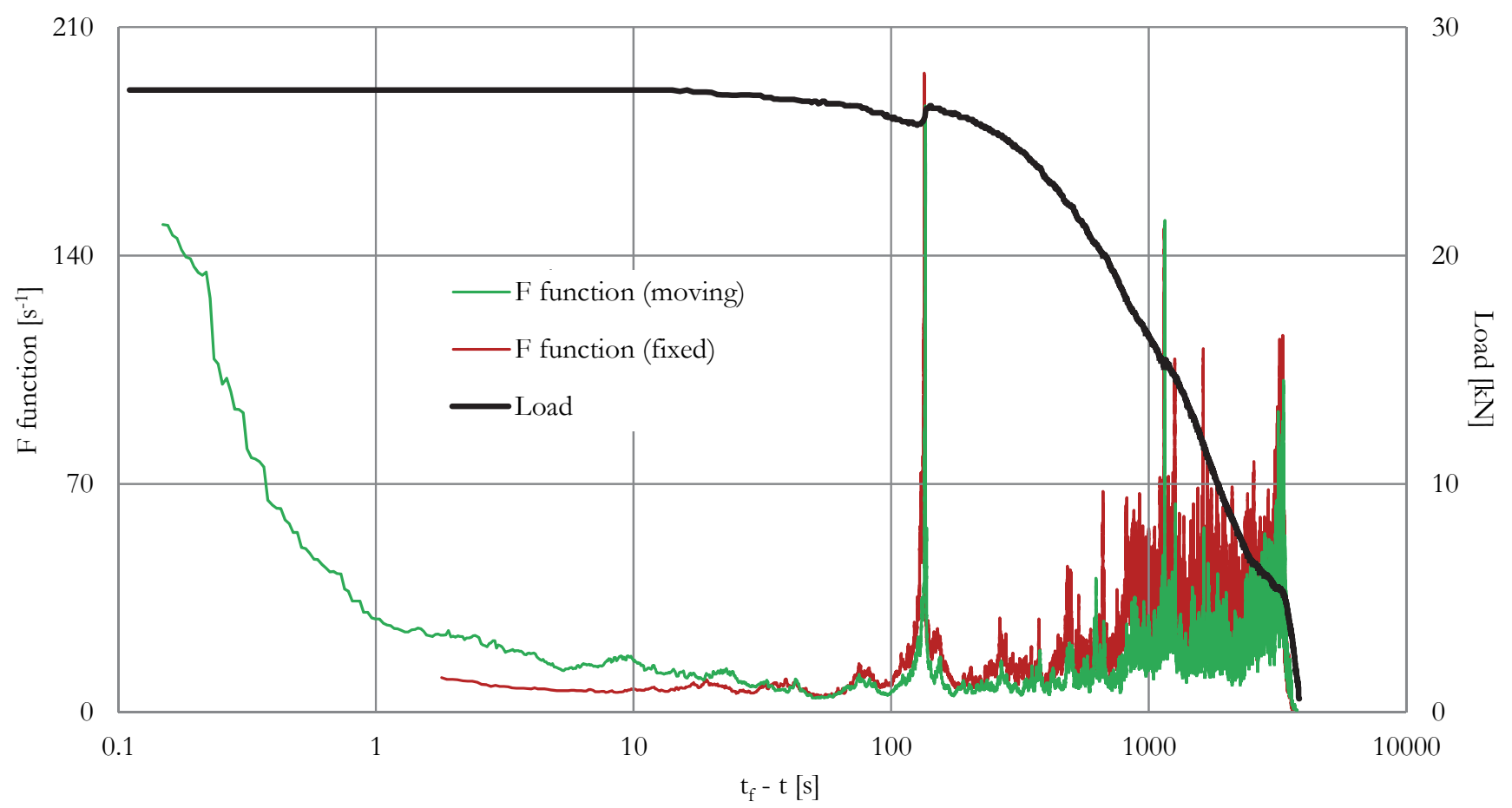

Figure 8: Semi-logarithmic plot of the F-function during the experiment of a typical specimen of Group B. 
Interesting information can be, also, obtained by studying the characteristics of the AEs rather than their number. Characteristics such as the average frequency ( $\mathrm{AF}$ ), the rise time and the amplitude are among the most useful ones. In Fig.9a the $\mathrm{AF}$ versus time of the experiment (of a Group B specimen) is plotted for two sensors, one attached on the moving volume and one on the fixed one. At a first glance, it can be seen that the values of the AF of the hits produced in the moving block are larger than the respective ones in the fixed volume, during the whole duration of the experiment. In addition, it is clear that the AF values of the hits in the fixed volume decrease smoothly until the end of the experiment. Similar behaviour is also exhibited by the hits of the moving block but only until the time instant of about $3000 \mathrm{~s}$. From this point on, the AF values gradually increase until the fracture of the specimen. On the contrary, the Rise Time per Amplitude (RA) values of the hits in the fixed volume are systematically larger than the respective values in the moving block (Fig.9b).

According to Grosse and Ohtsu [21], the combination of these two quantities can provide valuable information about the type of cracking modes occurred in the specimen. The categorization of the cracks can be, also, made based on the "AF versus Rise Time" plot [19], which is presented for typical Group A (Fig.10a) and Group B (Fig.10b) specimens of the main experimental protocol. Each marker corresponds to a loading phase of the specimen based on the slope changes observed in the respective load-displacement curves (Figs.3a). For both types of specimens, the cracks generated in the moving block are of tensile nature (green circles) contrary to the damage recorded in the fixed volume (red circles).
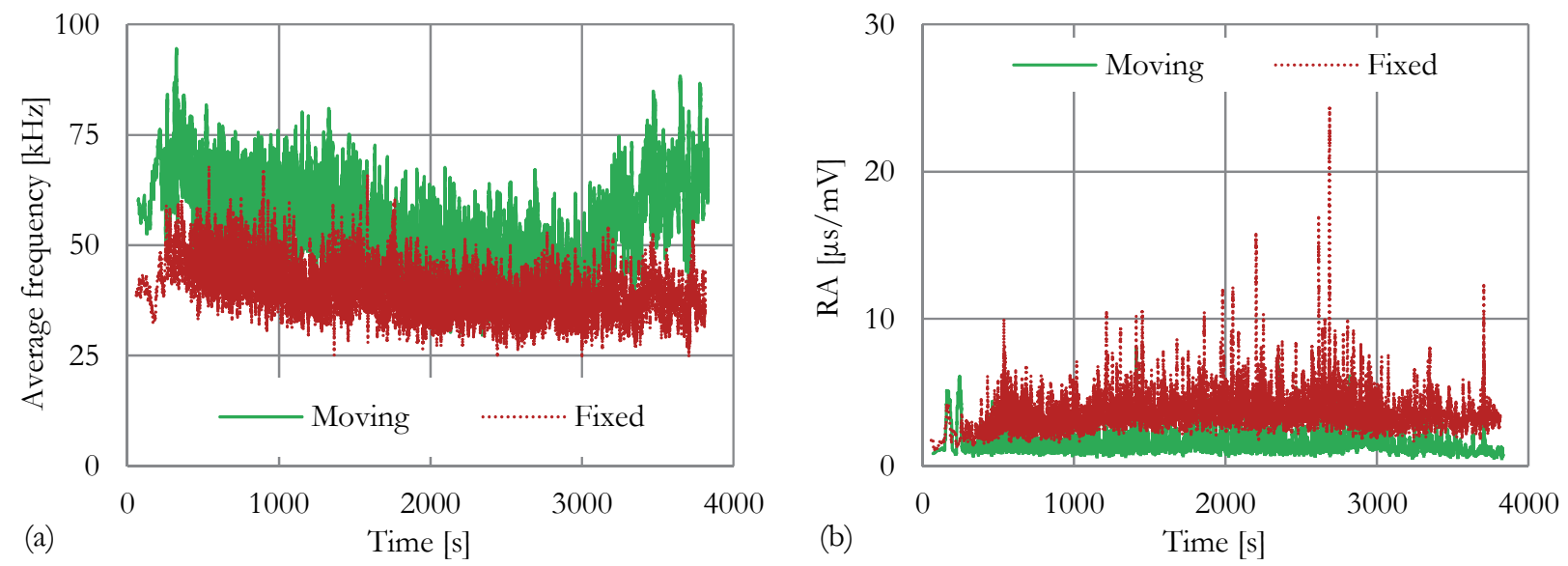

Figure 9: The temporal variation of the (a) average frequency and (b) RA parameter for a typical specimen of Group B.
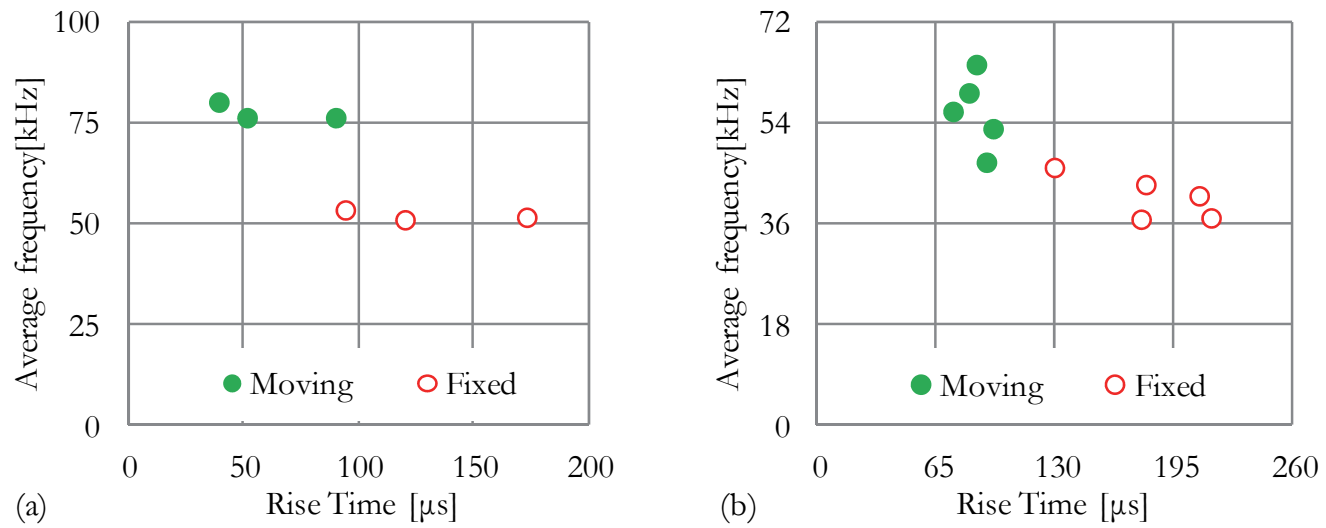

Figure 10: The Average Frequency (AF) versus the Rise Time for a typical specimen of (a) Group A and (b) Group B.

\section{The role of the Pressure Stimulated Currents (PSCs)}

Another technique used during the last years for monitoring the damage within the mass of a specimen is the Pressure Stimulated Currents (PSC) technique [22], which is based on the detection of very weak electric currents produced due to micro-fracturing. Besides monitoring damage it provides valuable warning signals of upcoming fracture [23]. One of the basic advantages of the PSC technique is the relatively simple and low cost equipment required, consisting of pairs of goldenplated electrodes and sensitive electrometers.

A single pair of electrodes was used in most of the experiments of the present study (attached on the front surface of the specimen) while two pairs of electrodes were used to only a few specimens (one pair on the front and one pair on the rear 
surface of the moving volume of the specimen). In Fig.11a the electric current recorded by both pairs during the loading of a specimen of Group B is plotted versus the applied load [24]. It is clear that the electric current detected in the moving volume (both on the front and on the rear surface of the specimen) is almost the same until a load-level of about $16 \mathrm{kN}$. After this load, the electric current on the rear part of the moving volume increases faster compared to what happens on the front part of the volume (which also increases but very smoothly) indicating the rear part of this marble volume as the more "active" one, i.e., with more severe damage accumulation.

In general, it can be stated that even one pair of electrodes records the electric activity (and therefore the damage evolution within the specimen [18]) according to a more or less satisfactory manner, assuming that it is attached close enough to the area of the expected failure. Unfortunately, this is not always known a priori, rendering the use of a second (or even more) pair of electrodes indispensable demand. In such a case (like the one discussed here) the different electric measurements recorded by each pair allows the determination of the area which is going to fail, even when complex specimens are loaded. The results are compatible with previously published ones [25] obtained when double edge notched marble plates were subjected to tensile loading. In those experiments, a pair of electrodes was attached close to each notch of the specimens and the electric current produced indicated the notch from which the crack was going to start. Very encouraging results were, also, obtained attaching a network of pairs of electrodes on prismatic marble specimens under compression [26].

The electric activity can be, also, studied in terms of the respective PSC energy using the equation:

$$
E_{P S C} \propto \int_{t_{i}}^{t_{i}+\Delta t} P S C^{2}(t) d t
$$

In Fig.11b, the temporal variation of the electric energy, recorded by the electrodes during an experiment of a Group A specimen, is plotted. The respective load-time curve is also shown in the same figure. The abrupt increase of the electric energy observed at $\mathrm{t} \sim 450 \mathrm{~s}$ and $\mathrm{t} \sim 700 \mathrm{~s}$ corresponds to the two slope changes of the load-time curve.
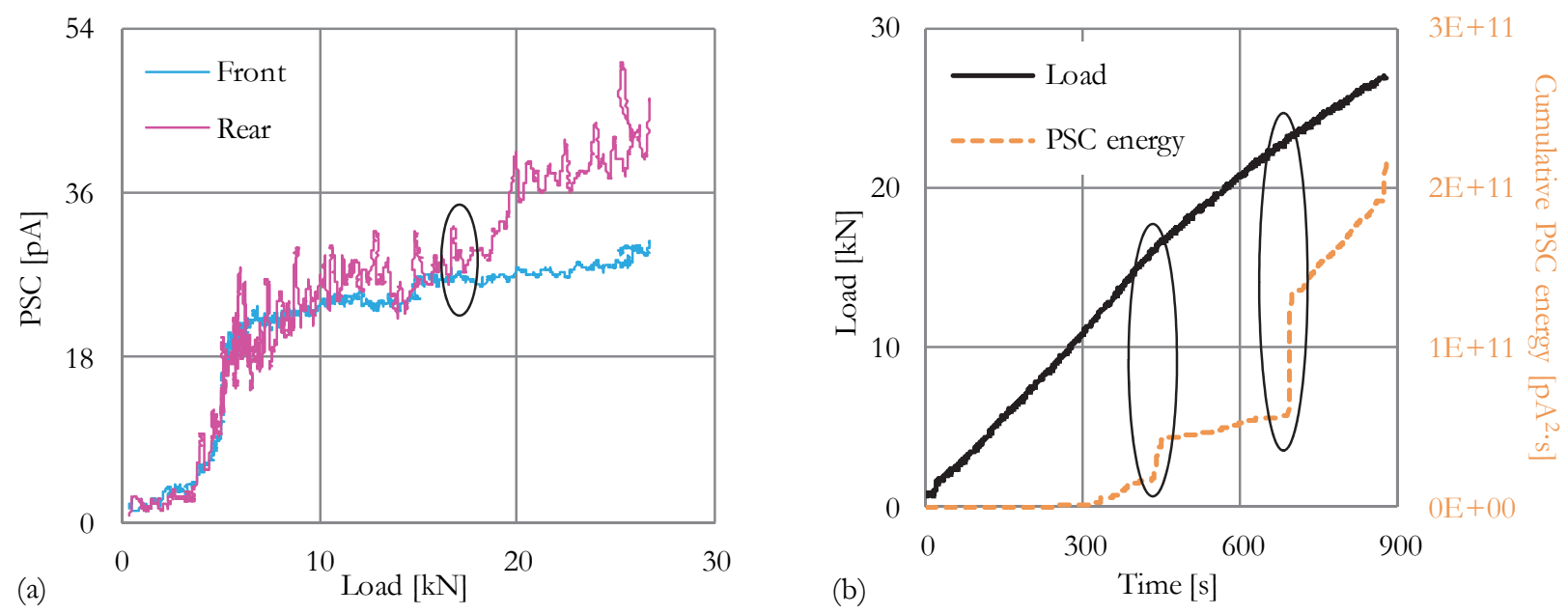

Figure 11: (a) The electric current recorded at the front and rear surfaces of a Group B specimen versus the applied load; (b) The temporal variation of both load and cumulative PSC energy of a specimen of Group A.

Plotting the PSC energy versus the respective mechanical energy, calculated as the area below the load-displacement curve (square points in Fig.12) for a typical specimen of Group A, a clear critical point is detected (black oval), i.e., a point at which a significant increase of the released energy is recorded. The same point is detected by the AE technique. It is known that in brittle materials the released energy recorded by the acoustic sensors is a measure of the size distribution of micro-cracks [27]. In Fig.12, the energy recorded by an acoustic sensor attached on the moving marble volume is also presented by triangles. The critical point is observed when the energy density equals about 26-29 J and the respective load level is equal to about $85 \%$ of the fracture load. This point corresponds to the last slope change observed in the load-displacement curve. The same procedure was followed by Pasiou and Triantis [28] in marble specimens under uniaxial compression and the critical point was determined at about $80 \%$ of the respective maximum stress, very close to the one obtained in the present study, although the specimens of the present study are complex (three materials exist, however marble is the one that is finally fractured) and the loading mode is different. 


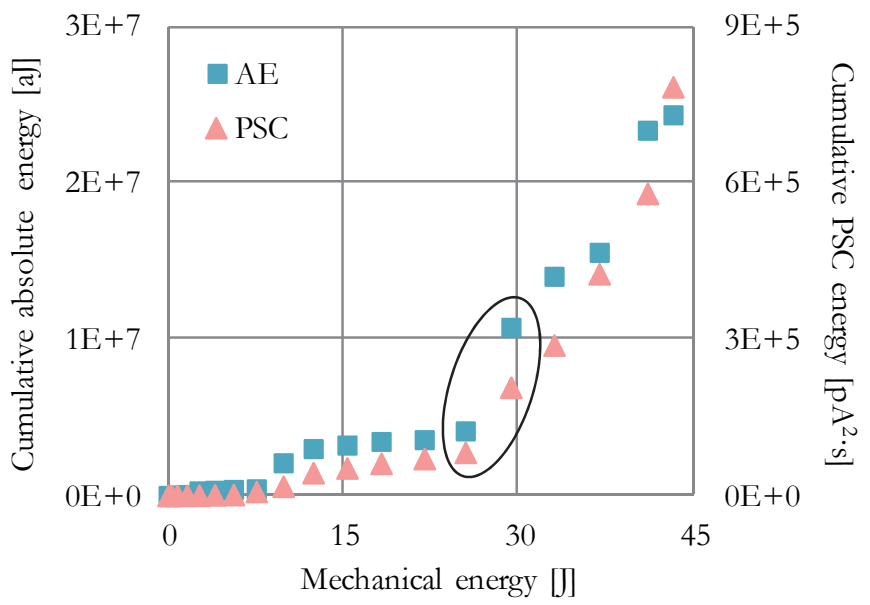

Figure 12: The released energy measured by AE and PSC techniques versus the mechanical energy for a typical specimen of Group A.

\section{CONCLUSIONS AND DISCUSSION}

he majority of the monuments of the classical Greek antiquity are mainly built using natural building stones, which are anisotropic and brittle materials. The mechanical response of such materials is usually characterized by the "size effect" [11-13], dictating the use of relatively large specimens with high cost, in case their behaviour is to be studied experimentally. The problem becomes more difficult when complex specimens, i.e., specimens made of more than one material, simulating structural members of monuments are studied. For such cases, standards/instructions concerning the geometry of the specimens or the experimental set-up do not exist. As a result, proper design of both the specimens' geometry and the experimental set-ups (according to the loading mode that is to be reproduced and the specific structural member that is going to be studied) becomes an one-way road.

Taking the above into account, it is easily understood that many restrictions are imposed concerning the basic equipment (especially the loading frame) that can be used for the implementation of such structural experiments. For example, the specimens must be smaller than the available space offered by the loading frame. At the same time, the loading frame must have the required capacity and also to be able to apply the load according to the required loading mode. In addition, proper equipment must be available by the frame otherwise a series of custom-made devices must be constructed.

As a second step and after the first class of difficulties (geometry, experimental set-up and equipment) is properly solved, the need for accurate measurements of various quantities arises. The raw data usually obtained during an experiment are the force and the displacement imposed by the loading frame on the specimen. For example, even in the case of a typical experiment (e.g., a standardized tensile test), the question concerning the accuracy of the displacement measured (or in other words of the deformation of the specimen) arises. For this reason, the elongation/strain of the specimen is usually obtained using external measuring instruments (like for example extensometers or strain gauges) and it is proven to be significantly different compared to the elongation/strain determined in terms of the displacement provided by the traverse of the loading frame. In addition, the measurements obtained by these traditional instruments refer to a specific point or a small area of the specimen. Moreover, the location at which they are attached is decided before the experiment and the researcher cannot obtain data from any other point/points of the specimen after the end of the experiment. Therefore, it is easily understood that in case of large specimens much more sophisticated sensing systems must be improvised. In this study the above difficulties were overpassed using the three dimensional DIC technique, which provides the actual displacement of the volume and permits the determination of the full-field displacement components.

In any case, the typical force-displacement curve (or stress-strain curve) accompanying every experiment is obtained. In case of a typical specimen subjected to a standardized test, the aforementioned curves are adequate to understand the mechanical behaviour of the specimen and their interpretation is quite simple. On the other hand, these typical curves can only roughly describe the mechanical behaviour of complex specimens and they are definitely not adequate for understanding the response of the specimen. In the present study, the combined use of the AE and the PSC techniques was catalytic since both of them not only monitored the internal damage developed in the specimen but they also located the area of the expecting fracture well before the fracture of the specimen occurs.

Summarizing the conclusions drawn during both the preliminary and the main stages of the experimental protocol (as well as during similar protocols carried out using the specific experimental techniques, i.e., 3D-DIC, AE and PSC), it is to be 
emphasized that all three of them are in excellent accordance to each other, despite the fact that the results obtained are of qualitative nature. This observation renders their use a "treasure" in the hands of researchers.

However, research in the laboratories should be somehow projected in real life. These techniques could be welcome for monitoring problematic members of a monument which will be always indicated by the scientists involved in the project, e.g., archeologists, architects, civil engineers etc. Taking into account the fact that a large number of structural members of most monuments are more or less cracked, these techniques could, for example, be used for monitoring existing cracks susceptible to propagate in order to alert the scientists about upcoming fractures in order to undertake the proper actions.

\section{REFERENCES}

[1] Livadefs, C. J. (1956). The structural iron of the Parthenon, J. Iron Steel I., 182, pp. 49-66.

[2] Varoufakis, G. J. (1992). The iron clamps and dowels from the Parthenon and Erechthion, J. Hist. Metall. S., 26, pp. $1-18$.

[3] Korres, M. and Bouras, Ch. (1983). Study for the Parthenon's Restoration, Athens, Ministry of Culture, Committee for the Conservation of the Acropolis Monuments.

[4] Angelides, S. (1976). Replacement of steel connectors by titanium alloy, The Acropolis: Problems-studies-measures to be taken. In: Proc $2^{\text {nd }}$ Int. Symp. on the Deterioration of Building Stones, Athens: National Technical University of Athens, pp. 351-352.

[5] Skoulikidis, Th. (1972). Deterioration des materiaux de construction et notamment des marbres par la corrosion de l' acier incorpore. Cas de l' Acropole. In: Proc 1st Int. Symp. on the Deterioration of Building Stones, Centre de Recherches et d' Etudes Océanographiques, La Rochelle, France: Les Imprimeries Reunies de Chambery, pp. 41-45.

[6] Skoulikidis, Th. (2000). Corrosion and Conservation of Structural Materials of Monuments, Crete, Crete University Press.

[7] Kourkoulis, S. K., Exadaktylos, G. E. and Vardoulakis, I. (1999). U-notched Dionysos-Pentelicon marble in three point bending: The effect of nonlinearity, anisotropy and microstructure, Int. J. Fracture, 98(3-4), pp. 369-392.

[8] Exadaktylos, G., Vardoulakis, I. and Kourkoulis, S. K. (2001). Influence of nonlinearity and double elasticity on flexure of rock beams - II. Characterization of Dionysos marble, Int. J. Solids Struct., 38, pp. 4119-4145.

[9] Kourkoulis, S. K., Prassianakis, I., Agioutantis, Z. and Exadaktylos, G. E. (2006). Reliability assessment of the NDT results for the internal damage of marble specimens, Int. J. Mater. Prod. Tec., 26(1/2), pp. 35-56.

[10] Zambas, C. (1994). Study for the Restoration of the Parthenon (vol. 3b). Athens: Ministry of Culture, Committee for the Conservation of the Acropolis Monuments.

[11] Vardoulakis, I., Exadaktylos, G. E. and Kourkoulis, S. K. (1998). Bending of marble with intrinsic length scales: A gradient theory with surface energy and size effects, J. Phys. IV, 8, pp. 399-406.

[12] Kourkoulis, S. K. and Ganniari-Papageorgiou, E. (2010). Experimental study of the size- and shape-effects of natural building stones, Constr. Build. Mater., 24(5), pp. 803-810.

[13] Kourkoulis, S. K. (2011). An experimental study of the mechanical behaviour of the 'Conchyliates' shell- stone: Some irregularities of the size effects, Strain, 47(S1), pp. e344-e356.

[14] Kourkoulis, S. K. and Ganniari-Papageorgiou, E. (2008). Bending of fragmented architraves restored with bolted titanium bars: A numerical analysis, Engineer. Transactions, 56(2), pp. 159-180.

[15] Kourkoulis, S. K., Ganniari-Papageorgiou, E. and Mentzini, M. (2010). Dionysos marble beams under bending: A contribution towards understanding the fracture of the Parthenon architraves, Eng. Geol., 115(3-4), pp. $246-256$.

[16] Christodoulopoulou, R. (2005). Study for the reposition of the members of the upper entablature of the Parthenon's northern peristyle, Athens, Ministry of Culture, Committee for the Conservation of the Acropolis Monuments.

[17] Kourkoulis, S. K., Pasiou, E. D., Triantis, D., Stavrakas, I. and Hloupis, G. (2015). Innovative experimental techniques in the service of restoration of stone monuments - Part I: The experimental set up, Procedia Engineering, 109C, pp. $268-275$.

[18] Triantis, D., Stavrakas, I., Pasiou, E. D., Hloupis, G. and Kourkoulis, S. K. (2015). Innovative experimental techniques in the service of restoration of stone monuments - Part II: Marble epistyles under shear, Procedia Engineering, 109C, pp. 276-284.

[19] Kourkoulis, S. K., Pasiou, E. D., Dakanali, I., Stavrakas, I. and Triantis, D. (2018). Notched marble plates under direct tension: Mechanical response and fracture, Constr. Build. Mater., 167, pp. 426-439.

[20] Triantis, D. and Kourkoulis, S. K. (2018). An alternative approach for representing the data provided by the Acoustic Emission technique, Rock Mech. Rock Eng., 51(8), pp. 2433-2438. 
[21] Grosse, C. U. and Ohtsu, M. (2008). Acoustic Emission Testing, Basics for Research - Applications in Civil Engineering, Heidelberg, Berlin, Springer-Verlag.

[22] Stavrakas, I., Triantis, D., Agioutantis, Z., Maurigiannakis, S., Saltas, V., Vallianatos, F. and Clarke, M. (2004). Pressure stimulated currents in rocks and their correlation with mechanical properties, Nat. Hazard. Earth Sys., 4, pp. 563-567.

[23] Anastasiadis, C., Stavrakas, I., Triantis, D. and Vallianatos, F. (2007). Correlation of pressure stimulated currents in rocks with the damage variable, Ann. Geophys., 50, pp. 1-6.

[24] Pasiou, E. D., Stavrakas, I., Triantis, D. and Kourkoulis, S. K. (2019). Marble epistyles under shear: An experimental study of the role of "Relieving Space", Frontiers Struct. Civil Eng., pp. 1-20.

[25] Kourkoulis, S. K., Pasiou, E. D., Dakanali, I., Stavrakas, I. and Triantis, D. (2018). Notched marble plates under tension: Detecting prefailure indicators and predicting entrance to the "critical stage", Fatigue Fract. Eng. M., 41, pp. $776-786$.

[26] Stavrakas, I., Kourkoulis, S. K. and Triantis, D. (2018). Monitoring spatiotemporal damage evolution in marble using electrical current and acoustic emissions, $1^{\text {st }}$ International Conference of the Greek Society of Experimental Mechanics of Materials, Book of Abstracts, Kourkoulis, S. K. and Triantis, D. (eds), ISSN: 2623-3541, pp. 197-198, Athens, Greece, May 10-12, 2018.

[27] Rao, M. V. M. S., Lakshmi, K. J. P., Rao, G. M. N., Vijayakumar, K. and Udayakumar, S. (2011). Precursory microcracking and brittle failure of Latur basalt and migmatite gneiss under compressive loading, Curr. Sci. (Bangalore), 101(8), pp. 1053-1059.

[28] Pasiou, E. D. and Triantis, D. (2017). Correlation between the electric and acoustic signals emitted during compression of brittle materials, Fract. Struct. Integrity, 40, pp. 41-51. 